\title{
Stellar populations in the highest redshift galaxies
}

\author{
Andrew J. Bunker \\ Department of Physics, University of Oxford \\ Denys Wilkinson Building, Keble Road, Oxford OX1 3RH, U.K. \\ email: Andy.Bunker@physics.ox.ac.uk
}

\begin{abstract}
I discuss stellar populations in galaxies at high redshift $(z>6)$, in particular the blue rest-frame UV colours which have been detected in recent years through near-IR imaging with HST. These spectral slopes of $\beta<-2$ are much more blue than star-forming galaxies at lower redshift, and may suggest less dust obscuration, lower metallicity or perhaps a different initial mass function. I describe current work on the luminosity function of high redshift starforming galaxies, the evolution of the fraction of strong Lyman- $\alpha$ emitters in this population, and the contribution of the ionizing photon budget from such galaxies towards the reionization of the Universe. I also describe constraints placed by Spitzer/IRAC on stellar populations in galaxies within the first billion years, and look towards future developments in spectroscopy with Extremely Large Telescopes and the James Webb Space Telescope, including the JWST/NIRSpec GTO programme on galaxy evolution at high redshift.
\end{abstract}

Keywords. galaxies: high-redshift, galaxies: evolution

Early work on the rest-UV colours of $z \approx 6$ Lyman break galaxies with NICMOS on the Hubble Space Telescope (HST) revealed blue spectral slopes of $\beta<-2$ (Stanway, McMahon \& Bunker 2005 MNRAS 359, 1184), much steeper than seen at lower redshift $(\beta \approx-1.5$ at $z \approx 2$, Reddy et al. 2010 ApJ 712, 1070). This finding was confirmed through later work with $H S T /$ WFC 3 (e.g., Wilkins et al. 2011, see Figures 1 \& 2). Such blue colours are inconsistent with average young dust-reddened stellar populations today, and imply that the metallicity and dust extinction were less at these high redshifts. This is consistent with the results from Spitzer/IRAC photometry in the rest-frame optical (Eyles et al. 2007 MNRAS 374, 910, see Figure 1), and also potentially explains the increase in the fraction of high-equivalent-width Lyman- $\alpha$ emission with redshift up to $z \approx 6$ (e.g., Stark et al. 2010 MNRAS 408, 1628, see Figure 2). At $z>7$ this fraction is seen to decline rapidly (e.g., Caruana et al. 2014 MNRAS 443, 2831, see Figure 2), probably due to the high neutral fraction at these redshifts quenching the Lyman- $\alpha$ emission line in the pre-Gunn-Peterson era. The reionization of the Intergalactic Medium (IGM) at $z \sim 8-10$ may be caused by star formation in early galaxies, but the currently-observed populations of Lyman break galaxies do not have sufficient photons to achieve this (Bunker et al. 2004 MNRAS 355, 374), suggesting that the bulk of the ionizing photons may come from galaxies below the current detection limits of $\mathrm{AB} \approx$ 29 mag (e.g., Lorenzoni et al. 2013 MNRAS 429, 150) due to a steep faint-end slope of the luminosity function. Future spectroscopy with NIRSpec on the James Webb Space Telescope will determine the true stellar continuum from Lyman break galaxies, and address the potential role of line contamination in the broad-band colours determined from HST and Spitzer imaging. Line ratios will also make direct measurements of the gas-phase metallicity and extinction of these galaxies, and this is a key objective in our 

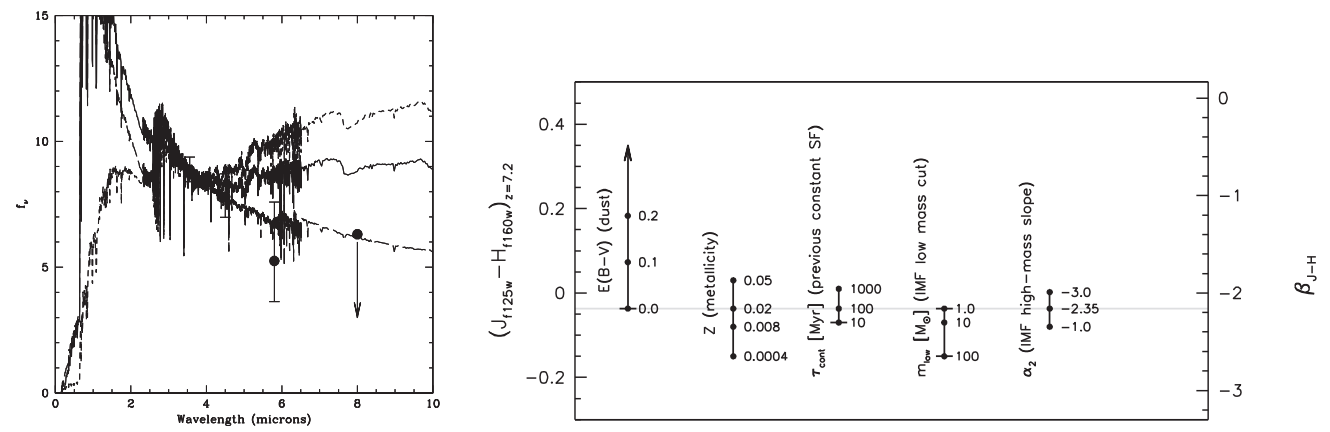

Figure 1. Left: Spitzer photometry of a stack of $i^{\prime}$-band drop-outs from GOODS-South (solid points are 3.6, 4.5, $5.8 \& 8 \mu \mathrm{m}$ ) which are uncontaminated by foreground sources from Eyles et al. (2007 MNRAS 374, 910). The curves are a $30 \mathrm{Myr}$ constant star-formation rate population synthesis model (Bruzual \& Charlot 2003) scaled to the same observed-frame $3.6 \mu \mathrm{m}$ flux density at $z \approx 6$, with solar metallicity (solid line), the same model with modest reddening of $E(B-V)=0.1$ (short-dash line) and no reddening and metallicity of $20 \%$ of solar (long-dash line). Only low metallicity and low extinction is compatible with the broad-band photometry.

Right: the effects of different factors on the restframe-UV continuum slope, $\beta$ (right axis) and observed $J-H$ colour in $H S T$ /WFC 3 (left axis) for $z \approx 7$ Lyman break galaxies from Wilkins et al. (2011 MNRAS 417, 717). The observed blue spectra slopes of $\beta<-2$ again suggest little dust and sub-solar metallicities, although other factors (such at the IMF) could also be responsible.
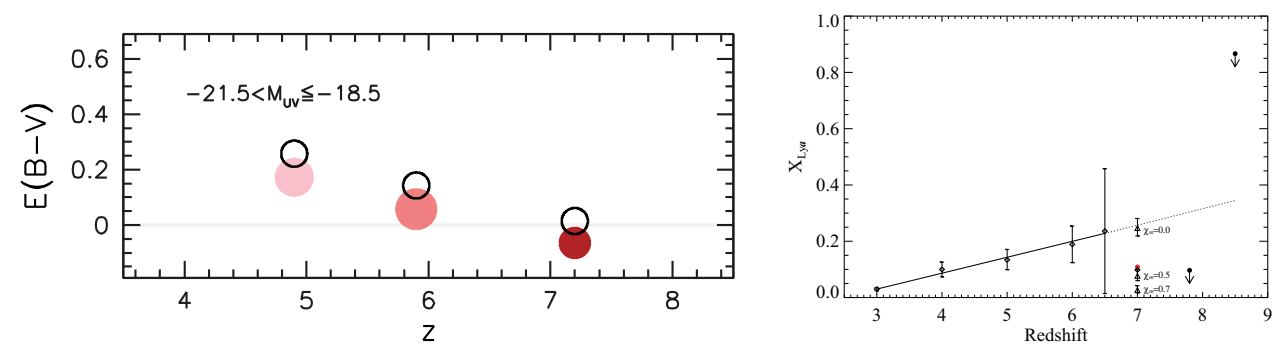

Figure 2. Left: the evolution of the restframe UV spectral slope from $z \approx 5$ to 7 (Wilkins et al. 2011 MNRAS 417, 717), expressed as the equivalent reddening $E(B-V)$ for a constant star formation rate $100 \mathrm{Myr}$ model with solar metallicity. The open circles denote the equivalent values inferred from the metallicity evolution in simulations.

Right: The fraction of high rest-frame equivalent width (EW $<75 \AA$ ) Lyman- $\alpha$ emission, including upper limits at $z \approx 7$ ( $z$-drops) and $z \approx 8.5$ ( $Y$-drops) from Caruana et al. (2014 MNRAS $443,2831)$. The diamond symbols at $z<7$ are results obtained at lower redshift by Shapley et al. (2003 ApJ 588,65) at $z=3$ and Stark et al. (2010 MNRAS 408, 1628) at $z=4-6.5$, and the trend seen of higher-EW with increasing redshift is extrapolated to higher redshifts (dotted line). Our upper limits at $z \approx 7.8$ would suggest that the IGM neutral fraction is $\chi_{\mathrm{HI}} \sim 0.5$.

NIRSpec Instrument Science Team guaranteed time observations (Jakobsen et al. 2012 BAAS 42, 396). 